\title{
Letter to the Editor: Resuscitative ultrasound - underappreciated need for the clarity in terminology
}

\section{Dear Editor,}

With pleasure, we read the manuscript of Dr. Becket ${ }^{1}$ that reports SHoC-ED2 data analysis and suggests that absence of cardiac activity on point-of-care ultrasound (POCUS) better predicts negative outcomes in cardiac arrest (CA) than electrocardiogram (ECG) alone. Agreeing that this report solidifies the evidence for POCUS use in resuscitative medicine, we notice the need for additional clarification surrounding the terminology of resuscitative ultrasound.

The term, resuscitative ultrasound, has been used in published literature in reference to three different concepts: 1) POCUS in $\mathrm{CA}^{1}$; 2) protocolized diagnostic POCUS in a hemodynamically unstable patient ${ }^{2}$; and 3) POCUS in fluid resuscitation management in shock. ${ }^{3}$ International Federation for Emergency Medicine recognized this problem and offered a position statement on sonography in hypotension and cardiac arrest (SHoC) that divides resuscitative POCUS into protocolized SHoChypotension and SHoC-CA. ${ }^{4}$ Although attempting to add clarity, by joining POCUS in CA together with the much larger field of POCUS in hemodynamically unstable patients, it actually limits the ability of resuscitative POCUS in CA to grow as a field in bedside ultrasonography.

Protocolized POCUS in hemodynamically unstable patients has been widely used, and mnemonics such as "RUSH" (rapid ultrasound

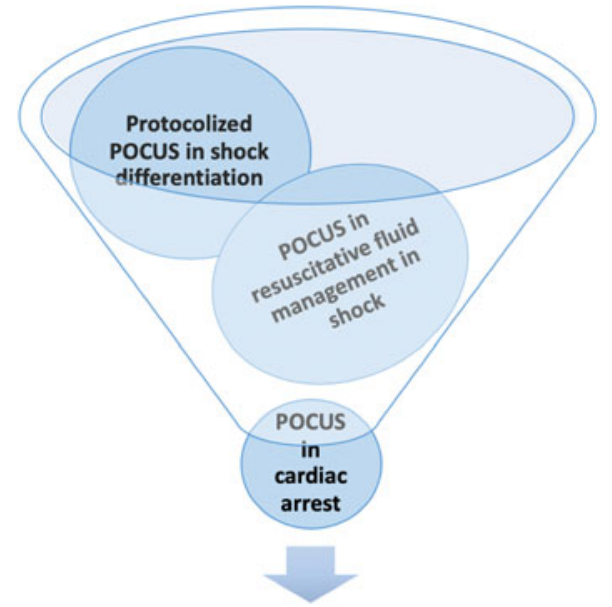

Resuscitative Ultrasound in shock) help avoid unnecessary diagnostic procedures or interventions in early management. The interest for the use of POCUS in fluid resuscitation in shock grew, along with recognition of the detrimental effect of fluid overresuscitation. Dynamic POCUS parameters offer guidance in fluid management and include cardiac output (CO) and left ventricular (LV) outflow tract Velocity Time Integral, ${ }^{5}$ corrected flow time variability, or inferior vena cava distensibility, ${ }^{3}$ allowing for more precise quantitative hemodynamic assessment and trending. In contrast, POCUS in cardiopulmonary arrest has been less systematically studied, although it has great potential as an additional comprehensive tool in advanced cardiovascular life support (ACLS). It can discriminate etiologies of the CA, identify reversible processes, aide with chest compressions, detect return of spontaneous circulation, and improve survival.

Appreciating the diversity and broad applicability of POCUS in hypotensive patients, we feel using the term "resuscitative ultrasound" as an umbrella term for all these concepts may not be appropriate. In its narrow sense, this term refers to the use of POCUS in CA, and we propose using the term primarily in reference to POCUS in CA that may offer clarity in terminology

Correspondence to: Dr. Igor Barjaktarevic, Division of Pulmonary and Critical Care, David Geffen School of Medicine at UCLA, 10833 Le Conte Avenue, CHS Building, Los Angeles, CA, 90095; Email: ibarjaktarevic@mednet.ucla.edu 
and allow this field to expand, as it deserves.

Igor Barjaktarevic, MD, $\mathrm{PhD}$

From ${ }^{*}$ Division of Pulmonary and Critical Care, Department of Medicine, David Geffen School of Medicine at UCLA, Los Angeles, California, USA

\section{Oren Friedman, MD}

${ }^{\dagger}$ Division of Cardiothoracic surgery, Cedars Sinai Medical Center.

Keywords: POCUS, cardiac arrest, resuscitation, ultrasound
Competing interests: Dr. Barjaktarevic reports grants and personal fees from GE Healthcare, outside the submitted work.

\section{REFERENCES}

1. Beckett N, Atkinson P, Fraser J, et al. Do combined ultrasound and electrocardiogram-rhythm findings predict survival in emergency department cardiac arrest patients? The Second Sonography in Hypotension and Cardiac Arrest in the Emergency Department (SHoC-ED2) study. CFEM 2019;21(6):739-43.

2. Perera P, Mailhot T, Riley D, Mandavia D. The RUSH exam: rapid Ultrasound in SHock in the evaluation of the critically lll [vii.]. Emerg Med Clin North Am 2010;28(1):29-56.

3. Lee CW, Kory PD, Arntfield RT. Development of a fluid resuscitation protocol using inferior vena cava and lung ultrasound. 7 Crit Care 2016;31 (1):96-100.

4. Atkinson P, Bowra J, Milne J, et al. International Federation for Emergency Medicine Consensus Statement: Sonography in hypotension and cardiac arrest (SHoC): An international consensus on the use of point of care ultrasound for undifferentiated hypotension and during cardiac arrest CORRIGENDUM. CFEM 2017;19 (4):327.

5. Bobbia X, Muller L, Claret PG, et al. A new echocardiographic tool for cardiac output evaluation: an experimental study. Shock 2019;52(4):449-55. 